\title{
Collision and phonation threshold pressures before and after loud, prolonged vocalization in trained and untrained voices
}

\author{
Laura Enflo, Johan Sundberg and Anita McAllister
}

\section{Linköping University Post Print}

\section{Tweet}

N.B.: When citing this work, cite the original article.

Original Publication:

Laura Enflo, Johan Sundberg and Anita McAllister, Collision and phonation threshold pressures before and after loud, prolonged vocalization in trained and untrained voices, 2013, Journal of Voice, (27), 5, 527-530.

http://dx.doi.org/10.1016/j.jvoice.2013.03.008

Copyright: Elsevier

http://www.elsevier.com/ 


\section{Collision and phonation threshold pressures before and after loud, prolonged vocalization in trained and un- trained voices}

Laura Enflo ${ }^{1,2}$, Johan Sundberg ${ }^{2}$, Anita McAllister ${ }^{1}$

${ }^{1}$ Dept. of Clinical and Experimental Medicine, Linköping University, Linköping, Sweden

${ }^{2}$ Dept. of Speech, Music \& Hearing, Royal Institute of Technology (KTH), Stockholm, Sweden

Corresponding author: Laura Enflo

Mailing address: KTH, Royal Institute of Technology, Dept. of Speech, Music and Hearing, Lindstedtsvägen 24, SE-10044 Stockholm, SWEDEN

Email address: lenflo@kth.se

Telephone number: +46702255335 


\begin{abstract}
The phonation threshold pressure (PTP) is defined as the lowest subglottal pressure needed for obtaining and sustaining vocal fold oscillation. It has been found to increase during vocal fatigue. In the present study, PTP is measured together with the threshold pressure needed for vocal fold collision, henceforth the collision threshold pressure (CTP). PTP and CTP are compared before and after loud, prolonged vocalization in singer and non-singer voices. Ten subjects repeated the vowel sequence /a,e,i,o,u/ at an SPL of at least $80 \mathrm{~dB}$ at $0.3 \mathrm{~m}$ for $20 \mathrm{~min}$. Audio and electroglottography signals were recorded before and after this exercise. At the same time, oral pressure was registered while the subjects produced a diminuendo repeating the syllable /pa:/, thus acquiring an approximate of the subglottal pressure. CTP and PTP increased significantly after the vocal loading in the non-singer subjects. On the other hand, singers reported no substantial effect of the exercise, and most singers had a mean after-to-before ratio close to 1 for both CTP and PTP.
\end{abstract}

Keywords: collision threshold pressure, phonation threshold pressure, electroglottography, vocal fatigue, vocal loading, prolonged vocalization, singing training, vocal training 


\section{Introduction}

Vocal loading is a term used to describe heavy voice use or phonotraumatic behaviors that may cause vocal fatigue. This vocal overuse has been suggested to be a repetitive strain injury resulting in tissue damage due to repetitive motion rather than an isolated event $(1,2)$. It may occur when the voice is used for an extended period of time without rest, perhaps also in a strenuous way. Important factors contributing to vocal loading are too loud vocalization, poor room acoustics and/or noisy environment, dry and/or polluted air, certain kinds of medication or liquids containing caffeine or alcohol, or dehydration (for a review, see e.g., 3). Vocal loading during work is regarded as an important factor frequently causing vocal fatigue and voice dysfunction. Some professions, e.g., teacher or aerobic instructor, are vocally very demanding (e.g., 4-10), and vocal problems have been observed to be overrepresented in school and pre-school teachers (e.g., 11-14). Although knowledge is extensive about what factors are likely to cause vocal problems, physiological effects of vocal loading have not yet been thoroughly studied and hence, there is still a need for objective measures of their consequences.

Many studies have shown that voice training can increase the ability to endure vocal loading (e.g., 15, 16). Yet, Heidel \& Torgerson (10) observed no significant difference between aerobics instructors with and without knowledge of vocal hygiene. However, the level of this knowledge was self-reported and unspecified.

Phonation threshold pressure (PTP), i.e. the lowest subglottal pressure $\left(\mathrm{P}_{\text {sub }}\right)$ causing and sustaining vocal fold vibration, tends to rise during vocal fatigue, even though the effect has been reported to be temporary $(17,18-20)$. Such a rise can be assumed to reflect an increase of vocal fold stiffness; stiffer vocal folds must be associated with a need for higher $\mathrm{P}_{\text {sub }}$.

Normally, phonation involves vocal fold contact during the vibratory cycle, but at pressures near PTP such contact mostly disappears. Vocal fold collision during phonation can be detected by means of an electroglottograph (EGG), since under such conditions conductivity across the glottis is high, causing high EGG amplitude. The lowest $\mathrm{P}_{\text {sub }}$ that initiates vocal fold collision has been referred to as collision threshold pressure, or CTP (21). Similar to PTP, CTP can be assumed to reflect vocal fold pliability. In a study of vocal warm-up in singers' voices, CTP was found to decrease after the exercise (21). McHenry and associates (22) found similar results for PTP. However, these effects on CTP and PTP reached significance only for the female subject groups. Also effects on CTP and PTP of resonance tube phonation with the tube end in water were recently studied in twelve female singers (23). Both CTP and PTP were found to rise, although only CTP significantly.

The aim of the present study is to examine the effects of loud, prolonged vocalization on CTP and PTP for singer and non-singer voices. We expect that (i) both CTP and PTP increase after the exercise, (ii) the CTP and PTP increase are greater for non-singer subjects than for experienced singers, and that (iii) non-singer subjects perceive phonation after vocal loading more strenuous than singer subjects do.

\section{Method}

\section{Experiment}

Ten subjects, two female (subject numbers 1 and 6) and eight male (subject numbers 2-5 and 7-10), volunteered as subjects. Subjects 6-10 were singers and had taken singing lessons; one of the nonsingers (3) had received some vocal training only as a child, while the remaining subjects all lacked vocal training. The subjects' age is listed in Table 1 . None of the subjects had any known voice problems at the time of the recordings. The task was to sing at least three diminuendo sequences of the syllable /pa:/, avoiding emphasis of the consonant /p/, starting at mezzo-forte, and continuing the sequence until voicing had ceased. This task was repeated three to six times on all pitches of an $\mathrm{F}$ major triad that fitted into their comfortable pitch range. The subjects were recorded in sitting position in a sound-treated booth. 
Table 1. Subject number, age, sex (male $\mathrm{M}$ or female $\mathrm{F}$ ) and number of years of formal voice training for all participants in the experiment.

\begin{tabular}{|c|c|c|c|}
\hline $\begin{array}{c}\text { Subject } \\
\text { Number }\end{array}$ & Age & Sex & $\begin{array}{c}\text { Formal } \\
\text { voice } \\
\text { training } \\
\text { [years] }\end{array}$ \\
\hline 1 & 30 & $F$ & - \\
\hline 2 & 27 & $M$ & - \\
\hline 3 & 35 & $M$ & - \\
\hline 4 & 32 & $M$ & - \\
\hline 5 & 45 & $M$ & - \\
\hline 6 & 28 & $F$ & 9 \\
\hline 7 & 73 & $M$ & $>10$ \\
\hline 8 & 18 & $M$ & 3 \\
\hline 9 & 22 & $M$ & 5 \\
\hline 10 & 18 & $M$ & 0.5 \\
\hline
\end{tabular}

The vocalization task was to phonate the vowel sequence /a,e,i,o,u/ at an SPL of at least $80 \mathrm{~dB}$ at $0.3 \mathrm{~m}$ during $20 \mathrm{~min}$ (24). One recording was made before and one immediately after the vocal exercise. After the recordings had been made, each subject was asked to describe how he/she felt when phonating after the vocalization, and whether or not he/she experienced vocal fatigue. The experimental design was approved by the regional ethical review board in Linköping.

Recordings were made of audio, oral pressure and EGG. Audio was picked up at $30 \mathrm{~cm}$ distance by a condenser microphone (B\&K 4003), with a power supply (B\&K 2812), set to $0 \mathrm{~dB}$ and amplified by a mixer, DSP Audio Interface Box (Nyvalla DSP). For subjects 1-7 oral pressure was recorded by means of a Gaeltec pressure transducer (Ltd, 7b) that the subject held in the corner of the mouth. As this transducer was unavailable at the time for the recordings of the remaining (male) subjects, oral pressure was obtained from a Glottal Enterprises pressure transducer (MSIF-2), attached to a thin plastic tube that which the subject held in the corner of his mouth. EGG was obtained from a two-channel electroglottograph (Glottal Enterprises EG 2), using the vocal fold contact area output and a low frequency limit of $40 \mathrm{~Hz}$. Contact gel was applied to improve skin contact if needed. During the recordings, EGG and oral pressure were monitored on an oscilloscope. Each of the three signals audio, EGG and pressure was recorded on a separate track of a computer by means of the Soundswell Signal WorkstationTM software (Core 4.0, Hitech Development AB, Sweden). Pressure from the Gaeltec transducer was calibrated by recording it while the transducer was immersed at a carefully measured depth in a glass cylinder with water. The Glottal Enterprises pressure transducer was calibrated by means of a manometer. The pressure values were announced on the recording.

\section{Analysis}

The analysis was performed using the Soundswell Signal Workstation. The oral pressure during the $/ \mathrm{p} /$ occlusion was accepted as an approximation of $\mathrm{P}_{\text {sub }}$. However, as this signal also contained some oral sound, it was LP filtered at $50 \mathrm{~Hz}$.

To reduce low frequency noise in the EGG signal it was HP filtered at $90 \mathrm{~Hz}$. The signal was then full-wave rectified, see Figure 1. Since absence of vocal fold contact produces a great reduction of EGG amplitude, loss of such contact can be identified. The subglottal pressures appearing immediately before and after a steep decrease of the full-wave rectified EGG amplitude were regarded as lying just above and just below the CTP. The average of these pressures was accepted 
as the CTP. For each subject and F0, CTP values were determined in at least three sequences and averaged. The same method of pressure determination was applied for PTP.

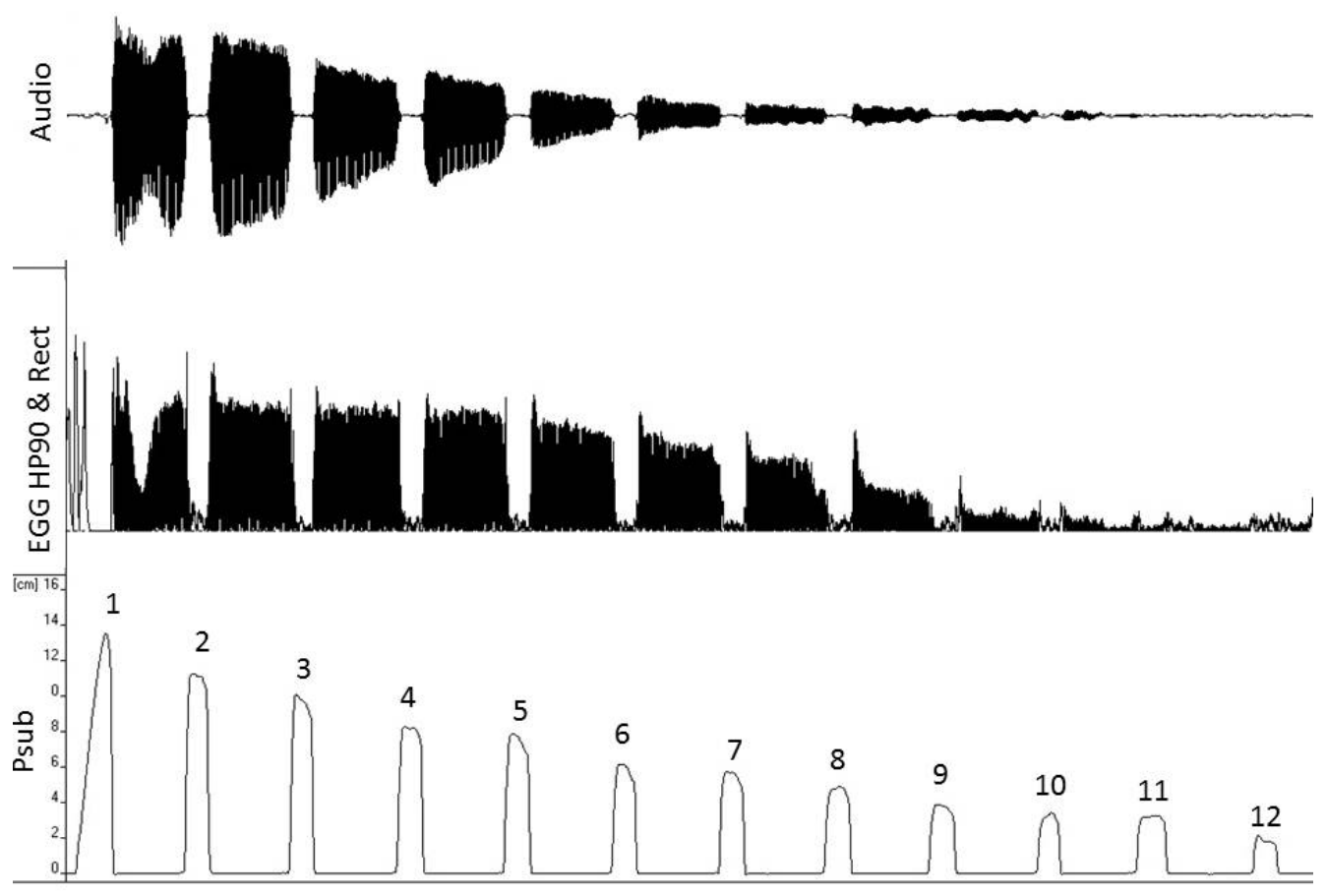

Figure 1: Example of the recordings analyzed showing the audio, the HP filtered and rectified EGG and the oral pressure signals (top, middle and bottom curves, respectively). The loss of vocal fold contact, reflected as a sudden drop in the rectified EGG signal amplitude, can be seen between pressure peaks 8 and 9. PTP, manifested as loss of phonation, occurred between pressure peaks 11 and 12 .

\section{Results}

All untrained subjects reported that they experienced vocal fatigue after the vocalization task. A majority of them showed higher CTP and PTP after the loading. The trained subjects, by contrast, reported that they experienced no fatigue after the exercise, except for subject 10, who had had no more than six months of vocal training.

Figure 2 shows examples of CTP and PTP for two subjects, one trained and one untrained. For the untrained subject, both thresholds tended to increase after the loading. For the trained subject, the exercise was not associated with any clear effect on the thresholds.

The effects of the vocalization task were evaluated in terms of the after-to-before ratio of both CTP and PTP. Table 2 lists the mean and SD across F0 of this ratio along with the number of F0 analyzed. CTP ratio varied between 1.64 and 0.78 and the corresponding variation of PTP ratio was 1.74 and 0.52 . For the untrained as well as for the trained subjects the CTP and PTP ratios, averaged across subjects, were similar. In the untrained subjects vocal loading caused a statistically significant increase of both CTP and PTP (paired samples t-test, $\mathrm{p}=0.000$ and $\mathrm{p}=0.001$, respectively). The trained subjects, by contrast, did not show any significant effects of loading, even though the ratio for the less well-trained subject 10 was rather large. 


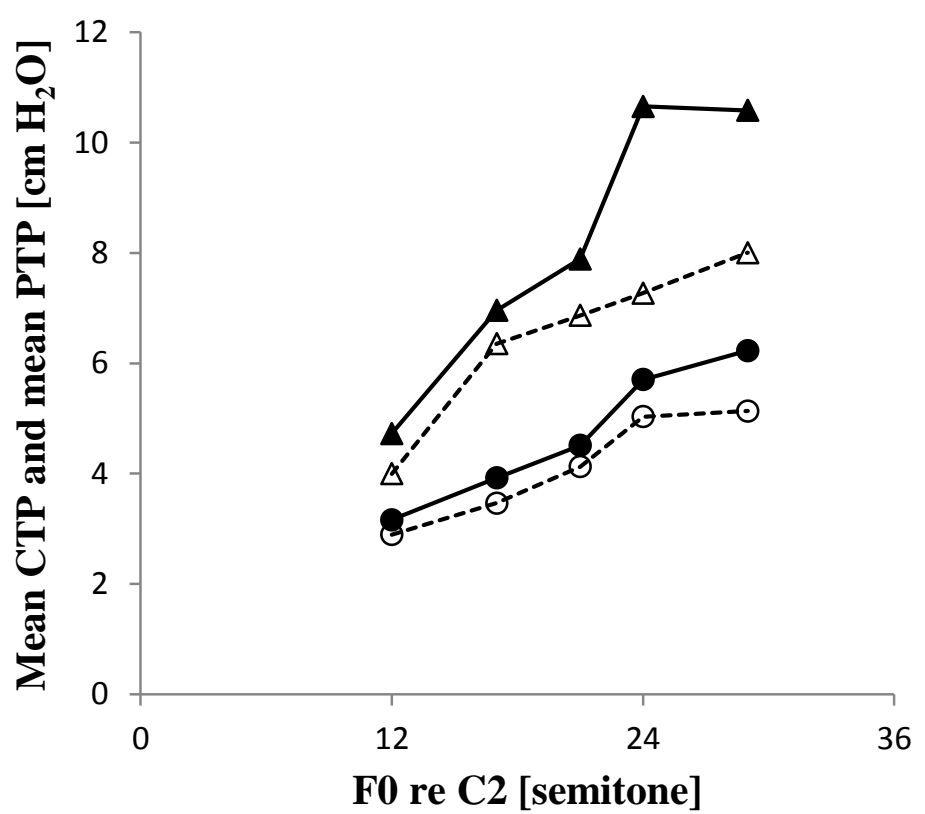

A

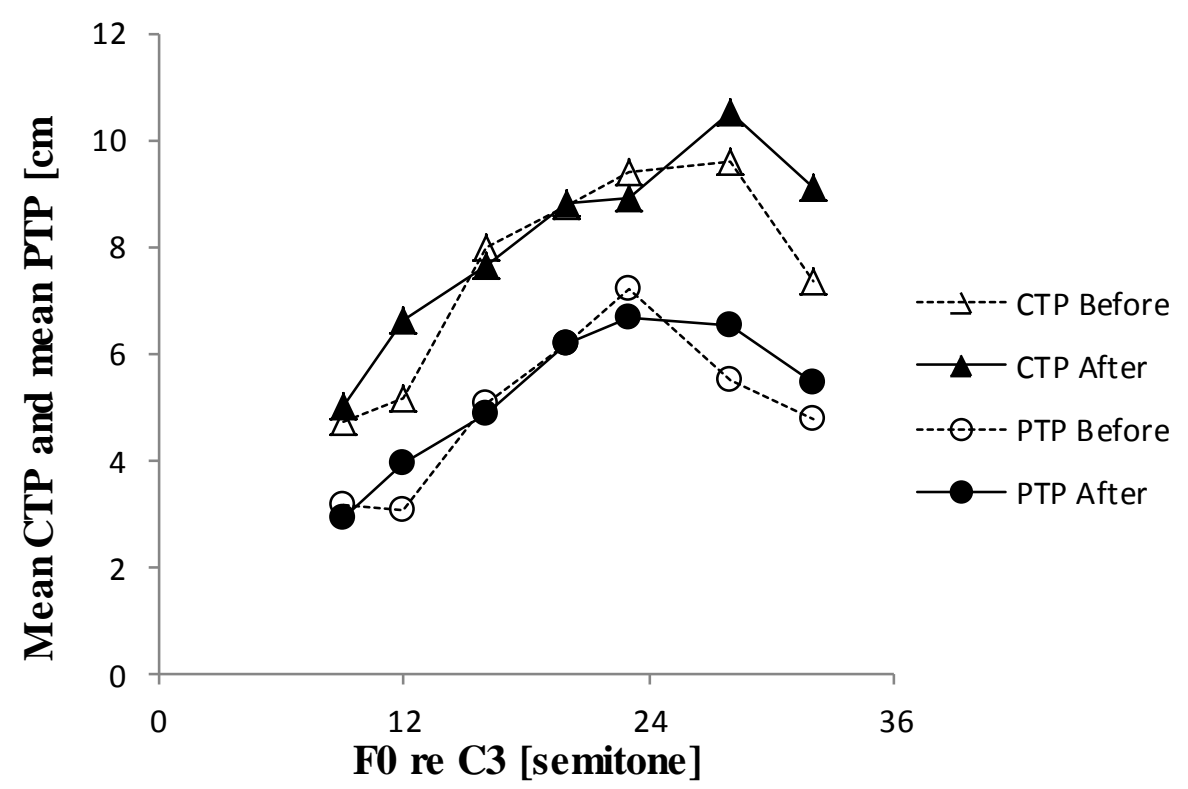

Figure 2: CTP and PTP values (triangles and circles) versus F0 in semitones relative to A) C2 (65.4 Hz) for untrained male subject 5 and B) C3 $(130.8 \mathrm{~Hz})$ for trained female subject 6. Dashed lines and open symbols and solid lines and filled symbols refer to threshold pressures before and after vocal loading, respectively. 
Table 2. Mean after-to-before ratio and SD across F0 for the CTP and PTP for the untrained and trained voices. $\mathrm{N}$ refers to the number of $\mathrm{F} 0$ analyzed.

\begin{tabular}{|cccccc|}
\hline & & \multicolumn{2}{c}{ CTP } & \multicolumn{2}{c|}{ PTP } \\
Untrained & $\mathbf{N}$ & Mean & SD & Mean & SD \\
1 & 2 & 1.49 & 0.14 & 1.62 & 0.07 \\
2 & 5 & 1.32 & 0.10 & 1.13 & 0.32 \\
3 & 5 & 1.06 & 0.04 & 1.02 & 0.11 \\
4 & 3 & 1.20 & 0.17 & 1.74 & 0.09 \\
5 & 5 & 1.24 & 0.15 & 1.13 & 0.05 \\
Mean & & $\mathbf{1 . 2 6}$ & $\mathbf{0 . 1 2}$ & $\mathbf{1 . 3 3}$ & $\mathbf{0 . 1 3}$ \\
Trained & & & & & \\
6 & 7 & 1.08 & 0.13 & 1.06 & 0.15 \\
7 & 5 & 1.07 & 0.13 & 0.98 & 0.03 \\
8 & 6 & 0.78 & 0.18 & 0.52 & 0.07 \\
9 & 5 & 0.86 & 0.29 & 1.19 & 0.29 \\
10 & 4 & 1.64 & 0.64 & 1.18 & 0.53 \\
Mean & & $\mathbf{1 . 0 9}$ & $\mathbf{0 . 2 7}$ & $\mathbf{0 . 9 9}$ & $\mathbf{0 . 2 1}$ \\
\hline
\end{tabular}

\section{Discussion}

The main finding of the present investigation was that for the untrained subjects and trained subject 10 the vocalization task caused a self-reported sensation of vocal fatigue and, as hypothesized for the untrained voices, also raised CTP and PTP significantly. The remaining four trained singer subjects, by contrast, reported that they did not experience any vocal fatigue, but rather an effect similar to that of vocal warm-up. Further, the trained singers showed minimal effects on CTP and PTP except in the case of singer 10, who had taken singing lessons for no more than six months. Small effects on CTP and PTP of the exercise were also observed in non-singer 3, who had sung in choir during childhood.

For the untrained voices, the exercise resulted in a rise of CTP and PTP, and all untrained subjects reported that they experienced vocal fatigue. On the other hand, it is not obvious that a rise of CTP is always associated with vocal fatigue. A significant rise of CTP was observed in twelve female singers after they had phonated for about two minutes into a "resonance tube", the end of which was submerged a few $\mathrm{cm}$ below the water surface (23). However, in that case, the subjects did not perceive any vocal fatigue, and voice experts tended to rate their voice quality as improved after the exercise. Hence, an elevated CTP alone is not a sufficient indicator of vocal fatigue; more reliable signs would be self-perceived vocal symptoms and voice quality changes.

This study supports the outcomes from several investigations that trained voices are less likely than untrained voices to be negatively influenced by loud, prolonged vocalization (e.g., 15, 16). Consequently, professions associated with high demands on the voice, such as school and preschool teachers, should have better possibilities to endure strenuous voice conditions if vocal training is provided as part of their professional education.

In this study, our aim was to analyze the effects of loud, prolonged vocalization in singers and non-singers. Our results indicated that the vocal loading exercise had an effect only in the nonsingers. This poses the question to what extent the singers' voices were actually loaded, and what type of exercise would have been needed to cause vocal fatigue in them. In any event, it probably would have been problematic to expose also non-singers to an exercise that would have caused vocal fatigue in singers.

Given our findings, it is tempting to speculate about physiological effects, which could have caused the rise of the CTP and PTP in the untrained subjects. It is clear that loud phonation requires elevated $\mathrm{P}_{\text {sub }}$, and it is possible that the untrained subjects performed the loading exercise with an increased glottal adduction. In other words, they may have changed their phonation type toward 
more hyperfunctional/pressed phonation. That would have increased the strain on the vocal fold tissues, which, in turn, could have made the folds stiffer and raised the CTP and PTP.

\section{Conclusions}

This investigation showed that loud, prolonged vocalization caused a significant increase of CTP and of PTP in untrained voices only. Singer subjects, by contrast, tended to perceive an effect similar to that of a vocal warm-up. Thus, the vocalization exercise used seemed to be insufficient to produce vocal fatigue in the trained singer voices, which apparently better endured the strenuous voice conditions.

\section{Acknowledgements}

The kind cooperation of the subjects is gratefully acknowledged. A preliminary version of this investigation was presented at the Interspeech conference in Brighton, UK, September 2009 (24).

\section{References}

1. Gray SD, Hammond E \& Hanson DF. Benign pathologic responses of the larynx. Ann Otol Rhinol Laryngol. 1995;104: 13-18.

2. Verdolini K, Rosen, CA \& Banski, RC. Classification Manual for Voice Disorders-I. Special Interest Division 3, Voice and Voice Disorders, ASHA. Lawrence Erlbaum Associates, Inc Publishers New Jersey; 2006.

3. Lehto L. Occupational voice - Studying voice production and preventing voice problems with special emphasis on call-centre employees. Ph.D. Thesis. Helsinki University of Technology, Laboratory of Acoustics and Audio Signal Processing; 2007.

4. Miller MK \& Verdolini K. Frequency and risk factors for voice problems in teachers of singing and control subjects. J Voice. 1995;9: 348-362.

5. Fritzell B. Voice disorders and occupations. Log Phon Voc. 1996;21: 7-12.

6. Mattiske JA, Oates JM \& Greenwood KM. Vocal problems among teachers: A review of prevalence, causes, prevention, and treatment. J Voice. 1998;12: 489-499.

7. Simberg S, Sala E, Vehmas, K \& Laine, A. Changes in the prevalence of vocal symptoms among teachers during a twelve-year period. J Voice. 2005;19: 95-102.

8. Lyberg Åhlander V, Rydell R \& Löfqvist A. Speaker's comfort in teaching environments: Voice problems in Swedish teaching staff. J Voice. 2011;25: 430-440.

9. Long J, Williford HN, Scharff Olson M \& Wolfe V. Voice problems and risk factors among aerobic intructors. $J$ Voice. 1998;12: 197-207.

10. Heidel SE \& Torgerson JK. Vocal problems among aerobic instructors and aerobic participants. J Commun Disord. 1993;26: 179-191.

11. Södersten M, Granqvist S, Hammarberg B \& Szabo A. Vocal behavior and vocal loading factors for preschool teachers at work studied with binaural DAT recordings. $J$ Voice. 2002;16: 356-371.

12. Vilkman E. Occupational safety and health aspects of voice and speech professions. Folia Phon Log. 2004;56: 220-253.

13. Laukkanen AM \& Kankare E. Vocal loading-related changes in male teachers' voices investigated before and after a working day. Folia Phon Log. 2006;58: 229-239.

14. Laukkanen AM, Ilomäki I, Leppänen K \& Vilkman E. Acoustic measures and self-reports of vocal fatigue by female teachers. $J$ Voice. 2008;22: 283-289.

15. Ilomäki I, Mäki E \& Laukkanen, AM. Vocal symptoms among teachers with and without voice education. Log Phon Voc. 2005;30: 171-174.

16. Lehto L, Alku P, Bäckström T \& Vilkman E. Voice symptoms of call-centre customer service advisers experienced during a work-day and effects of a short vocal training course. Log Phon Voc. 2005;30: 14-27. 
17. Titze I. Phonation threshold pressure: A missing link in glottal aerodynamics. $J$ Acoust Soc Am. 1992:91: 2926-2935.

18. Solomon NP \& DiMattia MS. Effects of a vocally fatiguing task and systematic hydration on phonation threshold pressure. $J$ Voice. 2000;14: 341-362.

19. Milbrath RL \& Solomon NP. Do vocal warm-up exercises alleviate vocal fatigue? J Speech Hear Res. 2003;46: 422-436.

20. Chang A \& Karnell MP. Perceived phonatory effort and phonation threshold pressure across a prolonged voice loading task: A study of vocal fatigue. $J$ Voice. 2004;18: 454-466.

21. Enflo L \& Sundberg J. Vocal fold collision threshold pressure: An alternative to phonation threshold pressure? Log Phon Voc. 2009;34: 210-217.

22. McHenry M, Johnson J \& Foshea B. The effect of specific versus combined warm-up strategies on the voice. $J$ Voice. 2009;23: 572-576.

23. Enflo L, Sundberg J, Romedahl C \& McAllister A. (in press) Effects on vocal fold collision and phonation threshold pressure of resonance tube phonation with tube end in water. To appear in J Speech Lang Hear Res.

24. Enflo L, Sundberg J \& Pabst F. Collision threshold pressure before and after vocal loading. In Proceedings of Interspeech. Brighton. 2009; 780-783. 